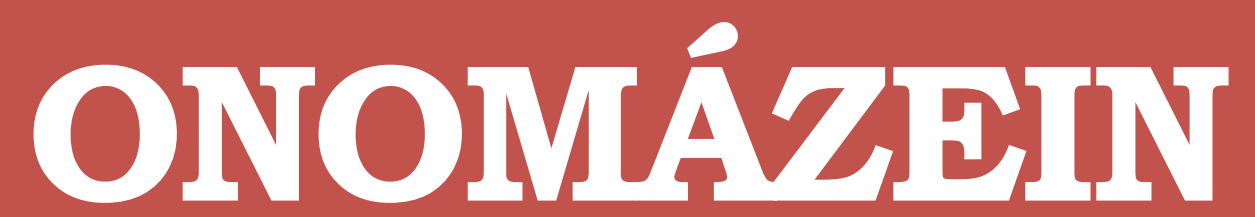

Revista de lingüística, filología y traducción
PONTIFICIA UNIVERSIDAD CATÓLICA DE CHILE FACULTAD DE LETRAS

Estudio sobre la traducción del léxico relacionado con la promoción oleoturística en Andalucía (español-alemán)

A study on the translation of the lexicon related to olive-oil tourism promotion in Andalusia (Spanish-German)

Número especial

- VII -

Discurso turístico, lenguas y traducción

2020
ONOMÁZEIN | Número especial VII - Discurso turístico, lenguas y traducción: 191-205

DOI: 10.7764/onomazein.ne7.11

ISSN: 0718-5758

\section{(c) $(\mathbb{i})$}

Alba Montes Sánchez: Departamento de Ciencias Sociales, Filosofía, Geografía, Traducción e Interpretación. Universidad de Córdoba. | E-mail:132mosaa@uco.es

Fecha de recepción: diciembre de 2019

Fecha de aceptación: abril de 2020 


\section{Resumen}

El oleoturismo ha surgido como una alternativa turística que tiene como objeto preservar los paisajes del olivar y promover el desarrollo rural y regional en comunidades autónomas como Andalucía, donde el aceite de oliva es uno de los principales motores económicos y un signo cultural y gastronómico. Para ello, la divulgación de esta nueva práctica turística a escala no solo nacional, sino también europea e internacional requiere de expertos en terminología y traducción que garanticen la correcta transferencia del conocimiento en esta área de especialidad. El presente estudio aborda el léxico y la terminología oleícola, empleada para comercializar la práctica del oleoturismo, desde una perspectiva traductológica, con el objetivo fundamental de analizarlos en el traslado del español al alemán y llevar a cabo una reflexión sobre los problemas y dificultades de traducción emergentes, como es el caso de los culturemas.

Palabras clave: aceite de oliva; culturemas; oleoturismo; problemas de traducción; terminología oleícola.

\section{Abstract}

Olive-oil tourism has emerged as a tourist alternative that aims to preserve the landscapes of the olive grove and promote rural and regional development in regions such as Andalusia, where olive oil is one of the main economic engines and a cultural and gastronomic sign. On this matter, the study of this new tourist practice not only nationally, but also at European and international level requires experts in terminology and translation that guarantee the correct transfer of knowledge in this area of expertise. The present study addresses the olive-oil lexicon and terminology used to commercialize the practice of olive-oil tourism, from a translational perspective, with the main objective of analyzing them in the transfer from Spanish to German and carrying out a reflection on the translation problems and difficulties found, as is the case of culturemes.

Keywords: olive oil; culturemes; olive-oil tourism; translation problems; olive-oil terminology. 


\section{Estado de la cuestión y planteamiento del estudio}

El oleoturismo ha sido abordado desde numerosos ámbitos de estudio, destacando principalmente aquellos relacionados con investigaciones sobre economía, gastronomía o historia (Agudo, 2010; Campón, Leco, Pérez y Hernández, 2010; Ruiz, Molina y Martín, 2011; Millán, Arjona y Amador, 2015; Elias y Barbero, 2017; Farré, Lozano y Aguilar, 2020). No obstante, los estudios relacionados con la traducción, la terminología o la lingüística aplicada son muy escasos en esta área. Si bien es cierto que existen investigaciones relacionadas con el aceite de oliva y la olivicultura desde un enfoque lingüístico (Montoro y Roldán, 2013; Ruiz Mezcua, 2017; El Ghalayini y Fendri, 2018; Medina y De las Montañas, 2019), no hemos encontrado estudios que establezcan un vínculo entre la traducción y el oleoturismo. Teniendo estos estudios como precedente, consideramos que la divulgación de esta nueva práctica turística a escala no solo nacional, sino también europea e internacional requiere de expertos en terminología y traducción que garanticen la correcta transferencia del conocimiento en esta área de especialidad. Por ello, para la realización de nuestro estudio partimos de la premisa de que la competencia traductora y el conocimiento de los aspectos lingüísticoculturales influyen de forma directa en la transferencia del conocimiento oleícola con fines turísticos y comerciales.

El presente estudio tiene como finalidad, por un lado, presentar una aproximación al concepto del oleoturismo, así como el desarrollo del turismo oleícola en la Comunidad Autónoma de Andalucía desde una perspectiva actual. Exponemos que el transvase de esta información del español al alemán implica una serie de problemas y dificultades, centrándonos en aquellas unidades fraseológicas denominadas culturemas. Para ello, a través del estudio de una guía publicada en español y traducida al alemán cuyo propósito es fomentar el turismo rural en la región sur de la provincia de Córdoba, presentaremos los culturemas más representativos relacionados con el oleoturismo, entre los cuales distinguimos aquellos que hacen referencia a construcciones y vivienda y los referidos a la gastronomía. De esta forma, la parte práctica de este estudio pretende realizar un análisis de los culturemas hallados en la terminología oleícola, la cual se emplea para comercializar la práctica del oleoturismo, desde un enfoque traductológico, con el objetivo fundamental de estudiarlos en español y en alemán y llevar a cabo una reflexión sobre las técnicas de traducción empleadas.

\section{Una aproximación al concepto "oleoturismo" en Andalucía}

La práctica del oleoturismo, también conocido como agroturismo, ha emergido como un área semejante al enoturismo, que ha logrado una alta potencialidad dentro del turismo rural en las últimas décadas. Según Moral y otros (2014: 30), algunos países europeos como Francia y Holanda fueron los principales propulsores de este tipo de turismo industrial que ha ido ganando terreno en un gran número de países de la Unión Europea y, por lo tanto, también en España se ha adentrado esta innovación turística. El entonces conocido como "turismo que 
gira en torno al mundo del olivo y el aceite de olivar" puede ser definido según Moral y otros (2014: 31) de la siguiente forma:

El oleoturismo, haciendo este concepto referencia a una novedosa práctica turística que tiene como motivación todo lo relacionado con el aceite de oliva y los recursos relacionados con el olivar, como pueden ser el agua, el terreno, así como otras especies de fauna y flora. Son por tanto elementos clave el clima, demanda turística, paisaje, a la hora de determinar la oferta y la demanda del oleoturismo, el cual nace bajo el exitoso ejemplo del enoturismo.

Millán y otros (2015: 202) delimitan una serie de actividades que se relacionan de forma directa con esta nueva concepción turística. En primer lugar, prima en el sector la contratación de visitas guiadas a almazaras, viejos molinos, haciendas, cortijos, oleotecas o museos del aceite con el objeto fundamental de conocer el proceso de producción del aceite de oliva. Existe también la opción de concertar visitas guiadas a las propias explotaciones agrarias de las principales zonas productoras para así apreciar el procedimiento y las técnicas empleadas en la recolecta del fruto. De hecho, se promueven rutas a pie, en bicicleta o en otro tipo de vehículos para conocer el terreno del olivar y disfrutar de la belleza paisajística. Según Ruiz y otros (2011: 92), es importante destacar que la creación y consolidación de una imagen turística se fundamenta en el estilo de vida y los aspectos culturales de sus habitantes, por lo que con esta práctica no solo se dan a conocer las novedosas prácticas tecnológicas por las que se produce el aceite de oliva, sino también los alojamientos rurales, los restaurantes y los pueblos oleícolas tradicionales.

Este tipo de turismo fomenta la inmersión del turista, generalmente un turista internacional, en las tradiciones y actividades de carácter cultural que giran en torno a las poblaciones oleícolas. Transmitir la cultura del olivo es uno de los propósitos fundamentales del oleoturismo, por lo que se promueven fiestas y ferias comerciales cuyo producto principal es el aceite de oliva. Estas celebraciones populares desarrolladas en ciertas localidades productoras presentan, además, experiencias gastronómicas con el AOVE y la aceituna como protagonistas.

\subsection{Andalucía como destino oleoturístico}

Con el fin de controlar los costes y optimizar la productividad, la innovación tecnológica ha desarrollado un rol fundamental. Según Moral y otros (2014: 30), el método de extracción conocido como "ciclo continuo" ha dibujado en el sector agrario un nuevo horizonte de alternativas que se caracterizan esencialmente por su intangibilidad. Estos nuevos ámbitos como la cosmética o el turismo se consideran intangibles ya que se definen como "aquellos activos no monetarios y sin apariencia física que se poseen para ser utilizados en la producción o suministro de bienes y servicios". Por tanto, en la actualidad, el sector oleícola en regiones como Andalucía ha evolucionado hasta presentar los denominados "intangibles del aceite de oliva”: oleoturismo, oleoecología, oleocultura, olivicultura, oleosalud y oleoeconomía. 
Desde hace años los organismos públicos y privados vienen trabajando en la elaboración de planes de fomento del turismo, entre los que destaca el III Plan de Calidad Turística de Andalucía 20142016, el cual tiene como objetivo general contribuir al desarrollo e impulso de un nuevo modelo de desarrollo turístico basado en criterios de calidad, accesibilidad y sostenibilidad en los destinos turísticos andaluces, promoviendo, al mismo tiempo, la innovación en la gestión turística y el apoyo a sectores turísticos poco consolidados que posibiliten la diferenciación y el liderazgo de Andalucía como destino de referencia internacional (Millán y otros, 2015: 198).

Según Millán y otros (2015: 195-196), preservar los paisajes del olivar y promover el desarrollo rural y regional en la Comunidad Autónoma de Andalucía son algunas de las cuestiones que encabezan las agendas políticas autonómicas y nacionales, sumadas al despoblamiento de las áreas de montaña, así como a "la crisis de la despoblación”, problema que inquieta cada vez más a la sociedad. El oleoturismo ha surgido como una alternativa turística que pretende hacer frente a estas preocupaciones económicas y sociales en Andalucía, donde el aceite de oliva es uno de los principales motores económicos y un signo cultural y gastronómico. De hecho, el oleoturismo se relaciona estrechamente con otras industrias emergentes andaluzas que emplean el aceite o hacen uso del olivo como elemento principal o como material de sus productos. Entre otras, podemos destacar firmas de cosmética, conservas o incluso artesanía de madera como partícipes de esta modalidad turística. Mientras que España, según Cañero y otros (2015: 136), ha sido configurada como el principal productor de aceite de oliva con certificación de calidad a nivel mundial, Andalucía se sitúa a la cabeza en explotaciones y producción oleícola, otorgando así al mundo del olivo y del aceite de oliva un carácter globalizado.

\subsection{La Guía turística Vía Verde del Aceite. Tramo de la provincia de Córdoba}

La Guía turística Vía Verde del Aceite. Tramo de la provincia de Córdoba (Reiseführer Vía Verde del Aceite. Abschnitt der Provinz Córdoba, en alemán) es un documento de promoción turística elaborado por el Patronato Provincial de Turismo de la Diputación de Córdoba y disponible en español, inglés y alemán. Dicha guía recopila la información del mayor atractivo turístico del sur de la provincia Córdoba sobre la Vía Verde del Aceite, que transcurre desde la provincia de Jaén hasta Puente Genil, municipio de Córdoba, contando con una extensión de más de 120 kilómetros. Entre los nueve municipios cordobeses abordados en la guía, destaca la riqueza patrimonial, las costumbres y tradiciones y la gastronomía, caracterizada por el aceite de oliva virgen extra de las Denominaciones de Origen Protegidas (DOP) de Baena y Lucena, así como la DOP Montilla-Moriles por sus aceites y vinos. Esto crea un enclave idóneo para la práctica del oleoturismo, pues engloba tanto alojamientos rurales (haciendas, caseríos o cortijos) como actividades y visitas (catas de aceite, visitas a molinos o almazaras) y degustaciones de platos típicos de estas zonas geográficas (la Campiña Este, la Campiña Sur y la Subbética), en las que el aceite de oliva es un ingrediente fundamental (mostachones, pestiños, salmorejo, gazpacho, tortas de aceite, etc.). Como toda guía turística, incorpora detalles sobre un itinerario específico, facilita alojamientos, direcciones, restaurantes, números de teléfono, direcciones, etc., acompañados de imágenes. En este caso en concreto, la guía se compone de 
100 páginas, lo que supone un total de 15920 vocablos en español. En su traducción al alemán, la suma de páginas es de 110 páginas, lo que supone un total de 15554 palabras.

\section{Problemas y dificultades de traducción en el lenguaje turístico promocio- nal: los culturemas}

Ortega (2016: 112) afirma que todos los individuos estamos condicionados por el "nicho evolutivo" en el que hemos crecido, condicionados por unos factores culturales que hacen difícilmente alcanzable la biculturalidad absoluta del traductor. Como consecuencia, la mencionada competencia intercultural del traductor puede verse opacada por esa biculturalidad parcial, haciendo que la traducción de textos turísticos, que suelen presentarse como textos multiculturales y multilingües, según González (2017: 38), sea una labor verdaderamente compleja. Esta actividad aborda campos como la geografía, la antropología o la economía, creando así diversas categorías de turismo en las que se engloban multitud de áreas de conocimiento: enoturismo, turismo de aventura, turismo rural, cicloturismo u oleoturismo, que es el tema central del presente estudio. Esta categoría presenta un léxico especializado, una terminología propia, la cual se enfrenta a problemas y dificultades en el proceso de conversión de la lengua de origen a la lengua meta, de la cultura de salida a la cultura de llegada.

Durán (2011: 522) plantea una distinción entre los problemas y las dificultades de traducción emergentes en los textos turísticos, definiendo los problemas como "intrínsecos del discurso turístico", es decir, propios de este tipo de discurso, que no se dejan influir por las circunstancias, mientras aclara que las dificultades traductológicas dependen enteramente del contexto en el que se enmarque la traducción, destacando así el encargo encomendado o las competencias del traductor, entre otros. De este modo, presenta la siguiente clasificación en problemas y dificultades de traducción:

\section{PROBLEMAS}

Subordinación de la traducción turística y falta de información

Frases ambiguas o de doble sentido

Culturemas

Ökosprache

Lenguaje positivo y poético ininterrumpido

Lenguaje confuso del TO

\section{DIFICULTADES}

Nombres propios de personas, instituciones, museos, etc.

Topónimos

Traducción inversa

Neologismos

Fuentes de referencia

\subsection{Los culturemas como problema de traducción}

A lo largo de la historia de la lingüística aplicada y la interrelación entre lenguas se han aportado multitud de definiciones diferentes para el término de "culturema". Según Molina (2006: 79), un culturema es "un elemento verbal o paraverbal que posee una carga cultural específica 
en una cultura y que al entrar en contacto con otra cultura a través de la traducción puede provocar un problema de índole cultural entre los textos origen y meta”. Estos fenómenos reflejados a través de palabras o expresiones son "léxico patrimonial" al poseer una gran carga cultural en la lengua, pues denotan elementos históricos, etnológicos, antropológicos, artísticos o sociales, entre otros.

Al igual que las diversas definiciones asociadas a este concepto, se han presentado numerosos apartados o ámbitos dentro de los cuales se pueden agrupar dichos signos culturales. Si seguimos la teoría de Katan (1999), se distinguen seis dominios: 1. Entorno (espacio, clima, vivienda o construcciones, vestimenta, alimentación o calendario); 2 . Conducta (normas de comportamiento); 3. Modos de comunicación; 4. Valores; 5. Creencias; 6. Identidad (engloba los cinco niveles previos).

Por tanto, en esta investigación analizamos el transvase del español al alemán de los culturemas que aparecen en un determinado contexto, detallando así las técnicas empleadas por el traductor para trasladar el contenido al TM. En este sentido, Molina (2006: 101) plantea un listado de técnicas de traducción de culturemas que está fundamentado en propuestas anteriores de otros estudios. De esta forma, para el análisis aplicado a la traducción que realizaremos a continuación nos guiaremos por el siguiente conjunto de técnicas de traducción:

$\begin{array}{ll}\text { Adaptación } & \text { Equivalente acuñado } \\ \text { Ampliación lingüística } & \text { Generalización } \\ \text { Amplificación } & \text { Modulación } \\ \text { Calco } & \text { Particularización } \\ \text { Compensación } & \text { Préstamo } \\ \text { Comprensión lingüística } & \text { Reducción } \\ \text { Creación discursiva } & \text { Sustitución } \\ \text { Descripción } & \text { Traducción literal } \\ \text { Transposición } & \text { Variación }\end{array}$

\section{Metodología del estudio y análisis de los culturemas orientado a la traducción}

Como hemos presentado con anterioridad, la Guía turística Vía Verde del Aceite. Tramo de la provincia de Córdoba (Reiseführer Vía Verde del Aceite. Abschnitt der Provinz Córdoba, en alemán) es un documento de promoción turística que recopila información sobre el atractivo turístico del sur de la provincia Córdoba, su riqueza patrimonial, sus costumbres y tradiciones y su gastronomía, que giran en torno al aceite de oliva. Con el propósito de analizar los culturemas presentes en este campo de especialidad, la metodología seguida se divide en tres pasos fundamentales, los cuales nos llevarán a extraer las conclusiones del presente estudio. 
Inicialmente hemos analizado de forma detallada el contenido incluido en el TO, haciendo especial hincapié en aquel léxico que se relaciona de forma directa con el oleoturismo: catas de aceite, degustaciones, cortijo-lagar, Museo del Olivar y del Aceite, visitas guiadas a almazaras, enoturismo, etc. Seguidamente, en aras de acotar el léxico extraído para el análisis de los culturemas, decidimos recurrir a la nivelación establecida por Katan (1999). Nos centramos, por tanto, en el primer dominio - entorno-y, a su vez, en aquellos culturemas que hacen referencia a construcciones o viviendas y a la gastronomía relacionadas con el aceite de oliva, la recolección de su fruto y su proceso de producción, así como su promoción turística y la de los diferentes productos y experiencias que tienen como fundamento el mundialmente conocido como "oro líquido". Por consiguiente, analizaremos los siguientes culturemas: almazara, lagar, cortijo, casería, hacienda, cortijada, aceitunas machacadas, salmorejo y empedraíllo. Así, analizamos lingüísticamente el verdadero significado de estos términos y su equivalente en alemán. Siguiendo las técnicas de traducción establecidas por Molina (2006), determinaremos qué procedimiento se ha seguido en la traducción del término. Igualmente, propondremos el uso de otra técnica si consideramos que la empleada no es del todo adecuada en dicho contexto, lo que nos Ilevará a presentar una serie de deducciones finales. Nuestro objetivo es dar cuenta del verdadero significado de los culturemas observados y analizar el trasvase de los mismos a la lengua alemana, todo ello sin olvidar que el contexto en el que se enmarcan presenta fines puramente turísticos.

\section{Almazara y lagar}

Una almazara es, según la RAE, un molino de aceite. Este término procede del árabe hispánico alma’șára, que procede a su vez del árabe clásico ma’șarah y significa 'lugar donde se exprime'. Dichas instalaciones se componen por lo general de tres espacios de trabajo que dejan diferenciar los diferentes procesos de producción: un patio de recepción, una nave de elaboración y una bodega. Cabe destacar que, de acuerdo con Bas (1981: 154), el término "almazara" se emplea principalmente en la región andaluza, dejando entrever la variedad existente en España dependiendo de la zona geográfica en la que se emplea. Por ejemplo, en Navarra, La Rioja o Aragón se emplea "trujal” para hacer referencia al molino de aceite, mientras que en Mallorca se denomina "tafona” y en la Comunidad Valenciana "almassera”. El TM ha empleado la técnica de la generalización para su traducción, proponiendo así en alemán el sustantivo Ölpresse. El equivalente correcto de dicho término sería "lagar”, mientras que para "almazara” es más apropiado el vocablo Ölmühle, ya que en su significado se especifica que los productos que se tratan son la aceituna y el aceite de oliva.

Si podemos tomarnos el viaje con calma, dedicando tiempo a realizar alguna de las visitas guiadas, o bien a participar en catas de vinos o aceites en las numerosas bodegas y almazaras que lo ofrecen, lo mejor es pasar alguna noche en Lucena, donde tenemos una amplia gama de alojamientos, para todos los gustos y bolsillos, entre los que elegir. 
TM Wenn man die Reise gelassen angehen kann, um Zeit mit irgendeiner Stadtführung oder einer Wein- oder Ölprobe in einer der vielen Bodegas und Ölpressen zu verbringen, übernachtet man am besten in Lucena.

Técnica Equivalente acuñado empleada

Según la RAE, un lagar es un "sitio donde se prensa la aceituna para sacar el aceite, o donde se machaca la manzana para obtener la sidra”, por lo que no se matiza si dicha instalación es para el tratamiento exclusivo de la aceituna, de la uva o de la manzana, produciéndose cierta ambigüedad. Entre otras de sus acepciones, la RAE expone que un lagar es la "tierra de poca extensión, plantada de olivar, y en la cual hay edificio y artefactos para extraer el aceite". Pese a dicha acepción en la que puede ser entendido como una almazara, el vocablo "lagar" generalmente hace referencia a aquellos edificios que trabajan la producción de la sidra o el vino. En la guía analizada podemos observar un ejemplo de ello, pues se emplea la técnica de la particularización para indicar que, en el edificio entendido como lagar, se produce vino, no aceite. De ahí que el término resultante en el TM sea Weinpresse, compuesto por los sustantivos Wein (vino) y Presse (lagar):

TO El moderno Centro de la Cultura del Vino se ha convertido en el epicentro del enoturismo en Moriles, organizando las visitas y catas que se realizan en muchos lagares y bodegas, así como en sus modernas instalaciones.

TM Das moderne Weinkulturzentrum ist zum Mittelpunkt des Weintourismus in Moriles geworden. Es organisiert die Führungen und Proben in vielen Weinpressen und Bodegas sowie in seinen modernen Einrichtungen.

Técnica Particularización

empleada

\section{Cortijo, casería, hacienda y cortijada}

Según la RAE, un "cortijo" se define como una "finca rústica con vivienda y dependencias adecuadas, típica de amplias zonas de la España meridional”. Para la RAE, “cortijo” proviene de corte, pero autores como Terencio y Marcos (1990: 69) van más allá y especifican que el término proviene del latín vulgar cohorticulum, diminutivo de cohors, lo que en español significa 'cohorte', 'patio' o 'corral'. La técnica utilizada para traducir este término al alemán es la particularización, puesto que al emplear el término Landhaus, compuesto por el sustantivo Land (campo) y el segundo sustantivo Haus (casa), se refiere de forma exclusiva a la construcción, no a todo el conjunto de la finca rural, tal y como especifica la definición ofrecida por la RAE.

TO Daremos nuestros últimos pasos o pedaladas siguiendo la huella del Tren del Aceite entre blancas erras salpicadas de verdes vides y olivos, y de algunos cortijos y bodegas. (81) 
TM Wir machen unsere letzten Schritte oder Pedaltritte auf der Spur der Olivenölbahn durch weiße Ländereien, die mit grünen Weinstöcken und Olivenhainen sowie ein paar Landhäusern und Winzereien versehen sind.

Técnica Particularización

empleada

Entre los diferentes tipos de cortijos, se puede distinguir la casería de olivar y la hacienda. El término "hacienda", definido como "finca agrícola", suele implicar una mayor monumentalidad. Aparece en el documento analizado como un topónimo (Hacienda Minerva), siendo, por tanto, traducido como tal. No obstante, para hacer referencia a este alojamiento se recurre al vocablo "cortijada”. Según Alcalá (1999: 173), "cortijada” deriva del término "cortijo" y, contrario a este, las "cortijadas" son propias de las zonas de minifundio y están integradas por pequeños núcleos de viviendas rurales que se rodean de tierras de cultivo. En este ejemplo se recurre a la técnica de la generalización, pues se traduce "cortijada" como Landgut (finca). No obstante, existe un equivalente acuñado en alemán para este término (Gruppe von Landgütern) porque estamos hablando de un conjunto de varios cortijos, no de una finca en particular.

TO Al poco, hallamos “Hacienda Minerva” (km 73,2), una magnífica cortijada rehabilitada con el sabor de lo tradicional, donde podemos alojarnos y deleitarnos con su preciosa biblioteca, su salón panorámico con chimenea, sus diversos comedores y terrazas.

TM Wenig später sehen wir die „Hacienda Minerva“ (km 73,2), ein traumhaftes renoviertes Landgut mit traditionellem Flair. Dort können wir uns eine Unterkunft nehmen und uns an der prächtigen Bibliothek, dem Salon mit Panoramablick und Kamin sowie den zahlreichen Speisebereichen und Terrassen erfreuen.

Técnica Equivalente acuñado

empleada

\section{Las aceitunas machacadas}

La aceituna (Olive) es el fruto del olivo del que se extrae el oro líquido. El país germano, aun siendo un país no productor, ha aumentado en un 73 \% su consumo de aceitunas de mesa $^{1}$, por lo que tanto el AOVE como la aceituna de mesa se han hecho un hueco en el estilo de vida de la sociedad alemana. Según el Real Decreto 679/2016, de 16 de diciembre,

1 Fuente: https://www.mapa.gob.es/es/agricultura/temas/producciones-agricolas/160427diagnost icoaceitunademesadefinitivo_tcm30-135524.pdf. Fecha de consulta: 12/02/2020. 
por el que se establece la norma de calidad de las aceitunas de mesa², se entiende por "aceitunas de mesa" lo siguiente:

Es el fruto de determinadas variedades del olivo cultivado (Olea europaea sativa Hoffg. Link.), sano, obtenido en el estado de madurez adecuado y de calidad tal que, sometido a las elaboraciones adecuadas previstas en el artículo 5, proporcione un producto listo para el consumo y de buena conservación.

En este ejemplo, analizamos la traducción de "aceitunas machacadas" (zerkleinerte Oliven), que se corresponde con un tipo de aceituna de color cambiante, pues se recoge antes de su completa madurez, y se somete a la preparación en aderezo. No obstante, existen diferentes formas de consumir la aceituna de mesa, entre las que se puede observar un fuerte componente cultural en su tratamiento, aliño y conservación del producto. En este caso, según su forma de presentación, se corresponde con aceitunas machacadas o partidas, que son aquellas "obtenidas de frutos enteros, sometidos a un proceso destinado a abrir la pulpa sin fracturar el hueso que permanece intacto y entero en el fruto". Dicha forma de presentación se diferencia de las rajadas (zerteilte Oliven) en que estas son "aceitunas con hueso con incisiones practicadas en la piel que afectan a parte de la pulpa". Asimismo, también se diferencian de las aceitunas encurtidas (eingelegte Oliven) en que estas, referidas como "alcaparrado", se presentan "con alcaparras y con material de relleno o sin él, envasadas con otros productos alimenticios, en las que las aceitunas predominen en comparación con el conjunto del producto comercializado en esta forma”.

Una vez establecidas estas distinciones siguiendo la legislación, observamos que en el proceso de traducción ha predominado la técnica de la traducción literal.

TO [...] entre otros muchos, y que podemos acompañar con un extenso repertorio de aceitunas (machacadas, encurtidas, rajadas,...) o alcaparrones.

TM [...] untervielen anderen, die wir begleitet von einem umfangreichen Repertoire an Oliven (zerkleinert, eingelegt, zerteilt usw.) oder Kapern genießen können.

Técnica Traducción literal

empleada

\section{Platos típicos: salmorejo y empedraíllo}

En cuanto a los culturemas que hacen referencia a la gastronomía y teniendo en cuenta que el texto que analizamos promueve la actividad oleoturística en Andalucía, destaca

2 Fuente: https://www.boe.es/boe/dias/2016/12/17/pdfs/BOE-A-2016-11953.pdf. Fecha de consulta: 04/01/2020. 
como plato estrella el salmorejo cordobés. El salmorejo es definido por la RAE como una "especie de gazpacho o puré frío a base de tomate, pan, aceite, ajo, y otros ingredientes machacados o batidos, propio de algunas zonas de Andalucía”. Según Martínez (2005: 365), el aceite constituye una de las combinaciones alimenticias más saludables, junto con otros elementos como el vino y los cereales, que integran la denominada "triología mediterránea". Por tanto, el aceite es, entre otros ingredientes recurrentes en la dieta mediterránea, un elemento esencial en la composición de delicias como el salmorejo, lo que hace que sea una comida a degustar en las experiencias gastronómicas propuestas en la guía. Con respecto al TM, la técnica de traducción empleada es el préstamo puro, pues se mantiene el vocablo sin ser modificado.

TO Verduras y frutas de sus huertas son convertidas en sabrosos platos como el pico salmorejo cordobés o el gazpacho, espinacas con garbanzos, naranjas picadas con bacalao, roñas de habicholones lucentinos [...].

TM Das Gemüse und Obst von den Plantagen werden zu leckeren Gerichten zubereitet, wie dem typischen Salmorejo Cordobés oder Gazpacho, Spinat mit Kichererbsen, gehackten Orangen mit Kabeljau, rostige grüne Bohnen [...].

Técnica Préstamo

empleada

El "empedraíllo" es uno de los guisos típicos del municipio de Baena, el cual se elabora con arroz, garbanzos, tomate, pimiento, cebolla, habichuelas, laurel, azafrán, sal, ajos y, por supuesto, aceite. Este plato de cuchara se compone de ingredientes de la zona, resultando en un puchero sabroso y consistente conocido de forma exclusiva en algunos municipios de la zona. En este caso, la traducción de este término al alemán implicaría emplear la técnica del préstamo, manteniendo la estructura, o la descripción, detallando que se trata de un guiso elaborado con arroz, garbanzos y otros productos de la región. No obstante, en el TM se ha optado por omitir dicho término, de modo que este plato se ha suprimido de la guía en versión alemana.

TO

TM

Técnica

empleada
Verduras y frutas de sus huertas son convertidas en sabrosos platos como el pico salmorejo cordobés o el gazpacho, espinacas con garbanzos, naranjas picadas con bacalao, roñas de habicholones lucentinos, las berenjenas fritas y el empedraíllo, entre otros muchos [...].

Das Gemüse und Obst von den Plantagen werden zu leckeren Gerichten zubereitet, wie dem typischen Salmorejo Cordobés oder Gazpacho, Spinat mit Kichererbsen, gehackten Orangen mit Kabeljau, rostige grüne Bohnen oder gebratene Auberginen, unter vielen anderen [...].

Omisión 


\section{Conclusiones}

A la hora de hacer un balance final, hemos confirmado la hipótesis inicial planteada en el presente estudio. Las tareas de documentación, el tratamiento del léxico y la terminología y la resolución de determinados problemas y dificultades de traducción emergentes durante el proceso de traducción están estrechamente ligados al conocimiento lingüístico-cultural. Como hemos evidenciado, en este sector no están implicados solo factores gastronómicos o turísticos, sino también culturales y económicos, por lo que es de crucial importancia trasladar la información y los matices culturales de forma adecuada. Por ello, se debe atender a la competencia lingüística de los profesionales que tratan con el léxico relacionado con el oleoturismo, en particular, y con otros tipos de turismo, en general, así como al conocimiento cultural en ambas lenguas de trabajo. En este sentido, el traductor debe ser bicultural.

En relación con la traducción de los culturemas en la Guía turística Vía Verde del Aceite. Tramo de la provincia de Córdoba, se han extraído una serie de reflexiones sobre algunas cuestiones fundamentales. En primer lugar, partimos de la premisa de que lengua y cultura son inseparables, ya que la lengua es elemento imprescindible de la cultura. Esto hace que la lengua sea la vía de transmisión cultural, lo que también se aplica a los textos de promoción oleoturística. En segundo lugar, hemos observado que para resolver este problema en el proceso de traducción se suele recurrir principalmente a las técnicas del préstamo, la generalización y la particularización. Consideramos que, al tratarse de elementos verbales con una gran carga cultural, no existen equivalentes acuñados que precisen tal información en la lengua de llegada, lo que nos hace reflexionar sobre la técnica de la descripción para este tipo de dilemas traductológicos. Si bien es cierto que muchos de estos casos se apoyaban en material gráfico para complementar la información, en la mayoría de los casos sería una buena alternativa describir el culturema que queremos trasladar.

Una apreciación más en relación con el léxico que estudiamos es la continua evolución lingüística a la que está sometido este sector y, más en concreto, el turismo rural. Es un hecho que la apuesta por destinos que se alejen del típico turismo de sol y playa está ganando terreno, fomentando el desarrollo de un turismo sostenible y ecológico, así como de su terminología. Esto, por tanto, supone que las diferentes vías de difusión y promoción de actividades y experiencias oleoturísticas, así como de comercialización de los productos derivados de dicho proceso, deben estar en constante evolución para adaptarse a la denominada "Ökosprache".

Como corolario, destacamos que la figura del traductor e intérprete actúa como mediador del texto oleoturístico al enfrentarse a la traducción de culturemas dentro de campos semánticos como la gastronomía, que tiene una fuerte presencia de estos elementos, seguido de las construcciones y viviendas. En este sentido, pretendemos que nuestro estudio enfocado en la traducción pueda transmitir los elementos que presentan carga cultural y que son muy 
comunes dentro del léxico analizado, lo que podría beneficiar tanto a los mediadores lingüísticos que traten con este tipo de textos como al desarrollo económico de aquellas comarcas que se dedican a la recolección, producción y comercialización del oro líquido andaluz, tesoro cultural de nuestra región.

\section{Bibliografía citada}

Agudo, Eva María, 2010: "Nuevas modalidades de turismo en un entorno rural: enoturismo y oleoturismo. Análisis del perfil del consumidor de turismo gastronómico", Universidad de Córdoba.

AlcalA, Antonio, 1999: Vocabulario Andaluz, Barcelona: El Mundo-Unidad.

BAs, Rosario de Fátima, 1981: "Contribución al estudio lingüístico de Cintruénigo (Navarra)", Fontes Linguae Vasconum 37, 99-170.

Campón, Ana María, Felipe Leco, Antonio Pérez y José Manuel Hernandez, 2010: "Agroturismo en Extremadura: una oportunidad para la diversificación económica de las áreas rurales" en Territorio, paisaje y patrimonio rural: Actas del XV Coloquio de Geografía Rural: Cáceres, 466-476.

Cañero, Pablo, Tomás López, Salvador Moral y Francisco Orgaz, 2015: "Análisis de la demanda del oleoturismo en Andalucía”, Revista de Estudios Regionales 2, 133-149.

DuRÁn, Isabel, 2011: El trabajo ontoterminográfico aplicado a la traducción de textos del turismo de aventura (español-inglés-alemán): fases de preparación, elaboración y edición. Tesis de maestría, Universidad de Málaga en Andalucía.

El Ghalayinı, Yara, y Mahdi Fendri, 2018: “Mecanismos metafóricos en el léxico de la cata de aceite de oliva en español”, Language Design 20, 41-56.

Elias, Silvina, y Andrea Cecilia BARBERo, 2017: "Situación del oleoturismo y lineamientos para su desarrollo en la región del sudoeste bonaerense, Argentina", RIAT - Revista Interamericana de Medioambiente y Turismo 13 (1), 91-104.

González, Diana, 2017: "Sobre las estrategias de traducción de referencias culturales en el ámbito de la arquitectura (español-inglés)”, Entreculturas 9, 37-52.

Farré, Marta, Carmen Lozano y Encarnación Aguilar, 2020: “La «nueva cultura del aceite» como eje de transformación en los territorios olivareros andaluces", AIBR: Revista de Antropología Iberoamericana 15 (1), 79-104. 
Katan, David, 1999: Traslating Cultures. An Introduction for Translators, Interpreters and Mediators, Manchester: St. Jerome.

Martínez, Francisca, 2005: "El vino en la medicina popular", Revista Murciana de Antropología 12, 368-372.

Medina, Ana, y Cristina de las Montañas, 2019: "Traducción de sitios web de empresas del sector del aceite de oliva: errores de localización" en Daniel Montañas (coord.): Nuevos estudios sobre traducción para el ámbito institucional y comercial, Suiza: Peter Lang: 55-77.

Millán, María Genoveva, Juan Manuel Arjona y Luis Amador, 2015: "El oleoturismo: una alternativa para preservar los paisajes del olivar y promover el desarrollo rural y regional de Andalucía - España”, Revista de Geografía Norte Grande 60, 195-214.

Molina, Lucía, 2006: El otoño del pingüino, Castelló de la Plana: Publicacions de la Universitat Jaume.

Montoro, Esteban T., y Mercedes Roldan, 2013: “Terminología, normalización y comunicación: Las categorías del aceite de oliva en español, inglés y chino”, Terminology. International Journal of Theoretical and Applied Issues in Specialized Communication 19 (1), 62-92.

Moral, Salvador, Pablo Cañero, Francisco Orgaz y Tomás López, 2014: “Una aproximación al oleoturismo en Andalucía, España”, International Journal of World of Tourism I (2), 29-40.

Ortega, Emilio, 2016: “De comunicación/incomunicación, traducción y cultura. Posibles aplicaciones al estudio interdisciplinar del espacio trasatlántico”, Transatlantic Studies Network, Revista de Estudios Internacionales 1, 111-119.

Real Academia Española (RAE) y Asociación de Academias de la Lengua Española (ASAlE), 2009: Nueva gramática de la lengua española. Morfología y sintaxis, Madrid: Espasa.

Ruiz, Aurora, 2017: Terminología y Traducción de Textos Agroalimentarios: Olivicultura (Inglés > Español), Córdoba: Ediciones Don Folio.

Ruiz, Ignacio, Valentín Molina y Víctor Manuel MarTín, 2011: "El oleoturismo como atractivo turístico en el medio rural español”, Papers de Turismo, 89-103.

Terencio, Marco, y Manuel-Antonio Marcos, 1990: De lingua Latina: edición bilingüe, BarceIona: Anthropos. 